\title{
On The Radio Antipodal Mean Number of Certain Types of Ladder Graphs
}

\author{
Kins Yenoke ${ }^{1}$, Arputha Jose $\mathrm{T}^{2}$, Venugopal $\mathrm{P}^{3}$ \\ Assistant Professor, Department of Mathematics, Loyola College, Chennai, India ${ }^{1}$ \\ Research Scholar, Department of Mathematics, SSN College of Engineering, Kalavakkam, Chennai, India ${ }^{2}$ \\ Associate Professor, Department of Mathematics, SSN College of Engineering, Kalavakkam, Chennai, India ${ }^{3}$
}

\begin{abstract}
Let $G=(V, E)$ be a graph and $\operatorname{diam}(G)$ be the diameter of the $G$. Let $u, v \in V(G)$. The radio antipodal mean labelling of graph $G$ is a function $f$ that assigns to each vertex $u$, a non-negative integer $f(u)$ such that $f(u) \neq$ $f(v)$ if $d(u, v)<\operatorname{diam}(G)$ and $d(u, v)+\left\lceil\frac{f(u)+f(v)}{2}\right\rceil \geq \operatorname{diam} G$, where $d(u, v)$ represents the shortest distance between any pair of vertices $u$ and $v$ of $G$. The antipodal mean number of $f$, denoted by $r_{a m n}(f)$ is the maximum number assigned to any vertex of $G$ and is denoted by $\operatorname{amn}(G)$. The antipodal mean number of $G$, denoted by $r_{a m n}(G)$ is the minimum value of $r_{a m n}(f)$ taken over all antipodal mean labeling $f$ of $G$. In this paper, the radio antipodal mean number for certain ladder-related graphs - Mongolian tent graph, Triangular ladder graph and Pagoda graphs were obtained.
\end{abstract}

KEYWORDS: Radio antipodal labeling, Radio antipodal mean labeling, Ladder graphs, Diameter of graph.

\section{INTRODUCTION}

Graph labeling is an assignment of integers to the vertices or edges or both, subject to some constraints. The labeling of vertices with non-negative integers is used to study the channel assignment problem introduced by Hale [14]. Here the vertices correspond to the radio transmitters and their labeling denotes the radio channel [13]. Gary Chartrand et.al. [5] introduced radio labeling, which is an extension of distance two labeling.

The antipodal labeling of a graph $G$ is a radio $k-1$ labeling such that, $d(u, v)+|f(u)-f(v)| \geq k$, for every distinct pair of vertices $u$ and $v$ of $G$. Determining the radio number of general graphs with diameter 2 is NP-hard and in general, its complexity is unknown [8]. The radio antipodal labeling was first introduced by Gary Chartrand in 2002 [6]. Khennoufa $\mathrm{R}$, and Togni $\mathrm{O}$ had given a note on the radio antipodal colouring of path [9]. The antipodal labeling of graphs like certain classes of Circulant graphs, cycles, some power of cycles, hexagonal mesh and grid were studied in $[2,4,7,11,12]$.

The radio antipodal mean labeling was introduced by Antony Xavier and Thivyarathi [3]. In their paper, they have studied the antipodal mean number of path, wheel and cycle. They have also completely determined the radio antipodal mean number for mesh and its derived architectures. Let $G=(V, E)$ be a graph and $\operatorname{diam}(G)$ be the diameter of the $G$. Let $u, v \in V(G)$. The radio antipodal mean labeling of graph $G$ is a function $f$ that assigns to each vertex $u$, a non-negative integer $f(u)$ such that $f(u) \neq f(v)$ if $d(u, v)<\operatorname{diam}(G)$ and $d(u, v)+\left\lceil\frac{f(u)+f(v)}{2}\right\rceil \geq \operatorname{diam} G$, where $d(u, v)$ represents the shortest distance between any pair of vertices $u$ and $v$ of $G$. The antipodal mean number of $f$, denoted by $r_{a m n}(f)$ is the maximum number assigned to any vertex of $f$ and it is denoted by $\operatorname{amn}(f)$. The antipodal mean number of $G$, denoted by $r_{a m n}(G)$ is the minimum value of $r_{a m n}(f)$ taken over all antipodal mean labeling $f$ of $G$.

In this paper, we have obtained the radio antipodal mean number of certain ladder related graphs - Mongolian tent graph, Triangular ladder graph and Pagoda graphs.

\section{Radio Antipodal Mean Number of Mongolian Tent Graph}

In this section, the bounds of radio antipodal mean number of Mongolian tent graph $M T(n)$ has been investigated.

Definition 2.1. [1]: The Ladder graph is a graph obtained by the Cartesian product of two path graphs $P_{2}$ and $P_{n}, n \geq 2$. The $\mathrm{n}^{\text {th }}$ dimension of a ladder graph has $2 n$ vertices and $3 n-2$ edges. It is denoted by $L_{n}$. 


\section{|| Volume 9, Issue 6, June 2020 ||}

Remark 1. The vertices of the ladder graph are labelled as $v_{i, j}, i=1,2,1 \leq j \leq n$.

Definition 2.2. [1]: The Mongolian tent graph is a graph obtained from the ladder graph $L_{n}$ by adding a new vertex $Z$ above the ladder and joining each vertex $v_{1, j}, 1 \leq j \leq n$ with $z$.

Remark 2. For our convenience, the vertices $v_{1, j}, 1 \leq j \leq n$ are considered to form the top row $R_{1}$ and the vertices $v_{2, j}, 1 \leq j \leq n$ forms the bottom row $R_{2}$ of $M T(n)$.

Lemma 2.1. The radio antipodal mean number of Mongolian tent graph MT(2)is 3.

Proof. The graph MT(2) is a planar graph with 5 vertices and 6 edges. The diameter of $M T(2)$ is 2 . See Figure 1.

Take $f(z)=1$. Label the vertex $v_{1,1}$ as 2 . Since the vertices $v_{1,1}$ and $v_{2,2}$ are diametrically opposite, by antipodal labeling condition, the vertex $v_{2,2}$ can also receive the same labeling as of $v_{1,1}$.

Label the vertex $v_{1,2}$ as 3 . As the distance between the vertices $v_{1,2}$ and $v_{2,1}$ is also equal to the diameter of $M T(2)$, the vertex $v_{2,1}$ also receives the same labeling as of $v_{1,2}$ as 3 .

Hence, $\operatorname{amn}(M T(2))=3$.

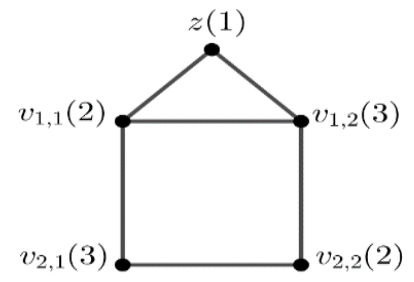

Fig. 1. Radio antipodal mean labeling of $M T(2)$

Lemma 2.2. The radio antipodal mean number of Mongolian tent graph $M T(3)$ is 5 .

Proof. MT(3)has 7 vertices and 10 edges and the diameter of MT(3) is 3. See Figure 2.

Label the vertices of $M T(3)$ as follows.

First label the single vertex $z$ as 1 . Then label the vertex $v_{1,1}$ as 2 . Since the vertices $v_{1,1}$ and $v_{2,3}$ are diametrically opposite, the vertex $v_{2,3}$ can also be labelled as 2 . Next label the vertex $v_{1,2}$ as 3 and $v_{1,3}$ as 4 . As the vertices $v_{1,3}$ and $v_{2,1}$ are at diametric distance, the vertex $v_{2,1}$ can also be labelled as 4 . The remaining vertex $v_{2,2}$ is labelled as 5 . It can be easily verified that in each case, the radio antipodal mean labeling condition is satisfied.

Hence, $\operatorname{amn}(M T(3))=5$.

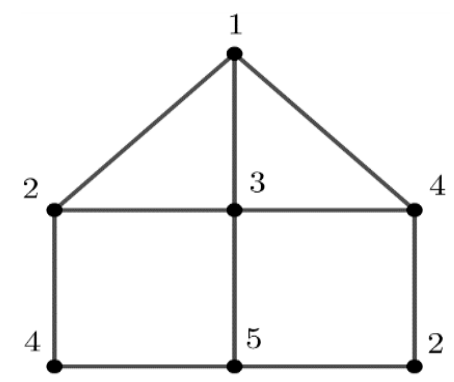

Fig. 2. Radio antipodal mean labeling of $M T(3)$

Lemma 2.3. The radio antipodal mean number of Mongolian tent graph $M T(4)$ is 7 .

Proof. The graph MT(4) has 9 vertices and 14 edges. Its diameter is 3 . See Figure 3.

The vertices of $M T(4)$ are labelled as follows. First, label the single vertex $z$ as 1 . The remaining vertices of $M T(4)$ are labeled by applying the antipodal mean labeling condition. Label the vertices $v_{1,1}, v_{1,2}, v_{1,3}$ and $v_{1,4}$ as $2,3,4$ and 5 respectively. The pair of vertices $\left(v_{1,1}, v_{2,4}\right)$ and $\left(v_{1,4}, v_{2,1}\right)$ are diametrically opposite to each other. Therefore the vertices $v_{2,1}$ and $v_{2,4}$ can be labelled as 5 and 2 respectively. The vertices $v_{2,2}$ and $v_{2,3}$ are labeled as 6 and 7 respectively. It can be easily verified that in each case, the radio antipodal mean labeling condition is satisfied.

Hence, $\operatorname{amn}(M T(4))=7$. 


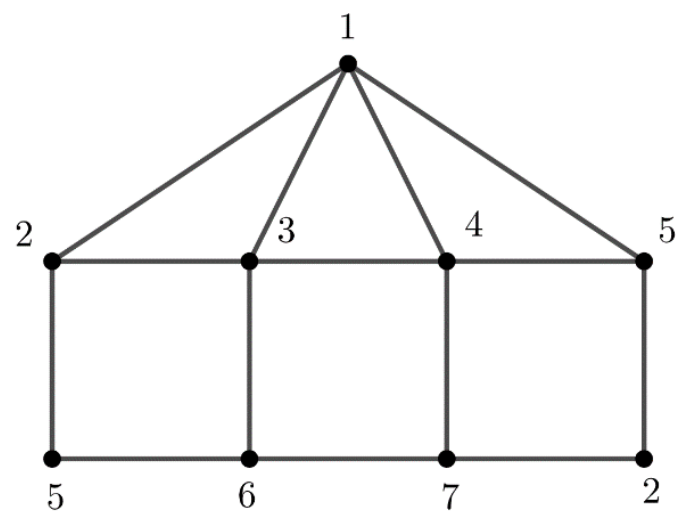

Fig. 3. Radio antipodal mean labeling of $M T(4)$

Theorem 2.1. $\operatorname{amn}(M T(n)) \geq n+5, n \geq 5$.

Proof. The graph $M T(n)$ has $2 n+1$ vertices. The diameter of $M T(n), n \geq 5$ is 4 .

The vertices in the bottom row of $M T(n)$ can be partitioned into 4 disjoint sets such that these vertices are at diametric distance. For instance, let $v_{a}=\left\{v_{2, a}: a \equiv 1 \bmod 4\right\}, v_{b}=\left\{v_{2, b}: b \equiv 2 \bmod 4\right\}, v_{c}=\left\{v_{2, c}: c \equiv 3 \bmod 4\right\}$ and $v_{d}=\left\{v_{2, d}: d \equiv 0 \bmod 4\right\}$ such that the vertices inside each set are internally disjoint set. See Figure 4.

It can be easily verified that, $v_{i} \cap v_{j}=\emptyset, i \neq j, 1 \leq i, j \leq 4$. All these $n$ vertices are at diametric distance 4 , hence they can be labelled with just 4 numbers. The remaining $n+1$ vertices of $M T(n)$ are not at diametric distance, hence at least $n+1$ numbers are required to label these vertices.

Hence minimum of $n+5$ numbers are required to label the vertices of $M T(n)$.

Therefore, $\operatorname{amn}(M T(n)) \geq n+5, n \geq 5$.

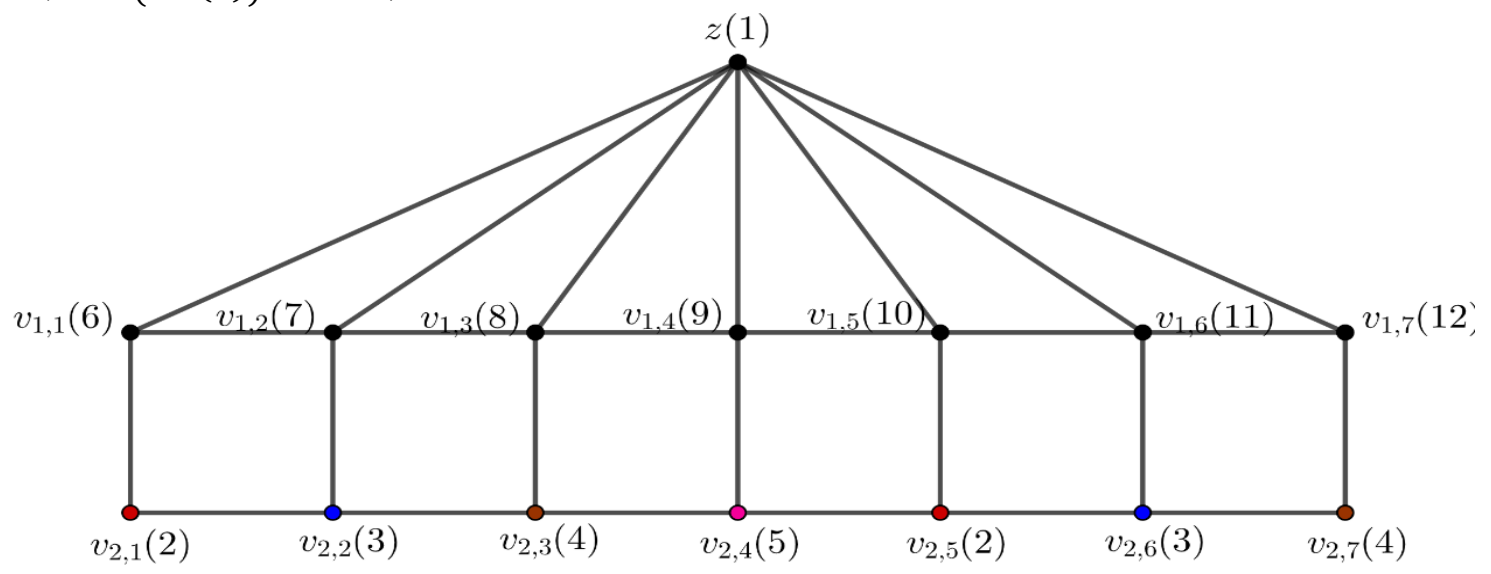

Fig. 4. Radio antipodal mean labeling of $M T(7)$

Theorem 2.2. $\operatorname{amn}(M T(n)) \leq n+5, n \geq 5$.

Proof. Let $\left\{z, v_{1,1}, v_{1,2}, \ldots, v_{1, n}, v_{2,1}, \ldots, v_{2, n}\right\}$ be the vertex set of $\operatorname{MT}(n)$.

Label the vertex $z$ as 1 .

The vertices in the top row of $M T(n)$ are labeled by the mapping,

$$
f\left(v_{1, j}\right)=j+5,1 \leq j \leq n
$$

And the vertices in the bottom row of $M T(n)$ are labelled by the mapping,

$f\left(v_{2, j}\right)=\left\{\begin{array}{l}2, \text { if } n \equiv 1 \bmod 4 \\ 3, \text { if } n \equiv 2 \bmod 4 \\ 4, \text { if } n \equiv 3 \bmod 4 \\ 5, \text { if } n \equiv 0 \bmod 4\end{array}, 1 \leq j \leq n\right.$

Claim: The mapping (1) and (2) are valid radio antipodal mean labeling. 
Let $u, v$ be any two vertices of $M T(n)$.

Case 1. Let the vertices $u, v$ lies in top row

Case 1.1. Suppose $d(u, v)=1$.

Then the vertex $u$ and $v$ will be of the form $u=v_{1, k}$ and $v=v_{1, k+1}, 1 \leq k \leq n$.

In this case, by mapping (1), $f\left(v_{k}\right)=k+5$ and $f\left(v_{k+1}\right)=k+6$ and $\left\lceil\frac{f\left(v_{k}\right)+f\left(v_{k+1}\right)}{2}\right\rceil=\frac{2 k+11}{2}$.

Hence, $d(u, v)+\left\lceil\frac{f\left(v_{k}\right)+f\left(v_{k+1}\right)}{2}\right\rceil \geq 1+\left\lceil\frac{2 k+11}{2}\right\rceil>4$.

Case 1.2. Suppose $d(u, v)>1$

Then the vertices $u$ and $v$ is of the form $u=v_{1, k}$ and $v=v_{1, k+a}, k \neq a, a+1,1 \leq a, k \leq n$.

By mapping (1), $\left\lceil\frac{f\left(v_{1, k}\right)+f\left(v_{1, k+a}\right)}{2}\right\rceil \geq\left\lceil\frac{2 k+a}{2}\right\rceil, 1 \leq k, a \leq n$ and $a \neq k, k+1$.

Therefore, in this case we haved $(u, v)+\left\lceil\frac{f\left(v_{k}\right)+f\left(v_{k+1}\right)}{2}\right\rceil \geq\left\lceil\frac{2 k+a}{2}\right\rceil>4$.

Case 2.Let the vertices $u, v$ lies in the bottom row.

Case 2.1.If the vertices $u, v$ lies in the same set, $u, v \in v_{2, j}, j=a, b, c, d$, then the vertices $u$ and $v$ will be at diametric distance. That is, $d(u, v)=4$ and the vertices $u$ and $v$ will be of the form $u=v_{2, j}$ and $v=v_{2, j}, j=a, b, c, d$.

By mapping (2), $\left\lceil\frac{f\left(v_{2, j}\right)+f\left(v_{2, j}\right)}{2}\right\rceil \geq 2$.

Therefore $d(u, v)+\left\lceil\frac{f\left(v_{2, j}\right)+f\left(v_{2, j}\right)}{2}\right\rceil>4$

Case 2.2. If the vertices $u$ and $v$ lies in two different set, $u \in v_{2, i}, v \in v_{2, j}, i \neq j, i, j=a, b, c, d$. Then $d(u, v) \geq 1$.

By mapping (2), $\left\lceil\frac{f\left(v_{2, i}\right)+f\left(v_{2, j}\right)}{2}\right\rceil \geq 3$.

Hence $d(u, v)+\left\lceil\frac{f\left(v_{2, i}\right)+f\left(v_{2, j}\right)}{2}\right\rceil \geq 4$.

Case 3: Suppose $u=z$ and $v=v_{i, j}, i=1,2$ and $1 \leq j \leq n$

Case 3.1: Let $v=v_{1, j}, 1 \leq j \leq n$.

In this case, $d(u, v)=1$

By mapping $(1), f(u)=1$ and $f\left(v_{1, k}\right)=k+5,1 \leq k \leq n$ and $\left|\frac{f(z)+f\left(v_{1, k}\right)}{2}\right| \geq 4$.

Case 3.2: Let $v=v_{2, j}, 1 \leq j \leq n$

In this case, $d(u, v)=2$.

By labeling (2), $\left\lceil\frac{f(u)+f(v)}{2}\right\rceil \geq 2$.

Hence, in all the cases it can be seen that $d(u, v)+\left\lceil\frac{f(u)+f(v)}{2}\right\rceil \geq 4$.

Therefore, (1) and (2) are valid radio antipodal mean labeling.

By mapping (1) the vertex $v_{1, n}$ receives the maximum label and is given by, $f\left(v_{1, n}\right)=n+5$.

Therefore, $\operatorname{amn}(M T(n)) \leq n+5$.

Theorem 2.3. $\operatorname{amn}(M T(n))=n+5$.

Proof. The proof is obvious from Theorem 2.1 and Theorem 2.2. 


\section{RAdio ANTIPOdal MeAn Number OF Triangular LADDER GraphS}

In this section, the upper bound of radio antipodal mean number for triangular ladder graph $T L G(n)$ has been obtained.

Definition 3.1. [1]: A Triangular ladder graph, denoted by $T L G(n)$, is a ladder graph obtained by adding the edges $\left\{\left(v_{i}, v_{\mathrm{n}+\mathrm{i}-1}\right), i=1,2, \ldots, n\right\}$. See Figure 5 .

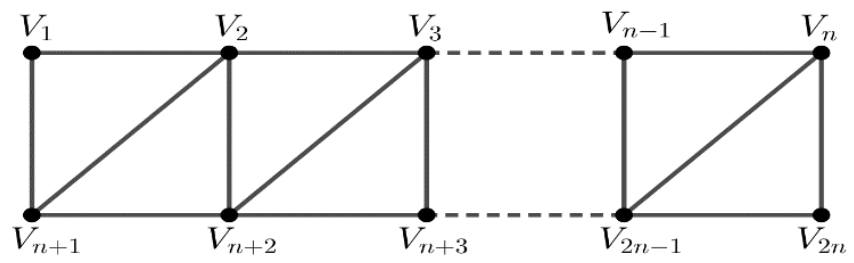

Fig 5. TLG(n)

Remark 2.TLG(n)has $2 n$ vertices and $4 n-3$ edges. Its diameter is $n$. The vertices $\left\{v_{1}, v_{2}, \ldots, v_{n}\right\}$ forms the top row $R_{1}$ and the vertices $\left\{v_{n+1}, v_{n+2}, \ldots, v_{2 n}\right\}$ forms the bottom row $R_{2}$ of $T L G(n)$.

Theorem 3.1. $\operatorname{amn}(T L G(2))=3$.

Proof. From Figure (6), TLG(2)has 4 vertices. Therefore, $\operatorname{amn}(T L G(2)) \geq 3$.

In $T L G(2)$, there is a pair of vertices at diametric distance. This implies, $\operatorname{amn}(T L G(2)) \leq 3$

Hence, $T L G(2)=3$.

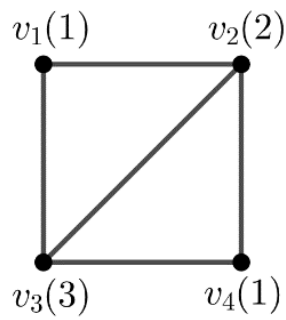

Fig. 6. $T L G(2)$

Theorem 3.2. The radio antipodal mean number of triangular ladder graph, $\operatorname{amn}(T L G(n)) \leq 2 n+k, k \equiv 0 \bmod n-$ $4, n \geq 3$.

Proof. Let $\left\{v_{1}, v_{2}, \ldots, v_{n}, v_{n+1}, \ldots, v_{2 n}\right\}$ be the vertices of $T L G(n)$. See Figure 7 .

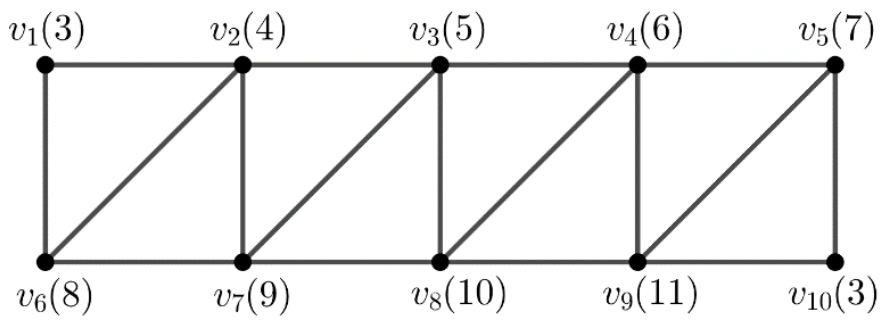

Fig. 7. TLG(5)with its antipodal mean labeling.

In these vertices $v_{1}$ and $v_{2 n}$ are at diametric distance and hence they receive the same labeling. Therefore, $f\left(v_{1}\right)=$ $f\left(v_{2 n}\right)$.

The remaining $2 n-1$ vertices of $T L G(n)$ are labeled by the mapping,

$$
f\left(v_{i}\right)=n+i-3,1 \leq i<2 n
$$




\section{| e-ISSN: 2319-8753, p-ISSN: 2320-6710| www.ijiirset.com | Impact Factor: 7.512|}

\section{|| Volume 9, Issue 6, June 2020 ||}

Claim: The mapping (3) is a valid radio antipodal mean labeling.

Let $u, v$ be any two vertices of $T L G(n)$.

Case 1: Suppose $u$ and $v$ are not extreme vertices $\left(v_{1}, v_{n}, v_{n+1}\right.$ and $\left.v_{2 n}\right)$

Clearly, $d(u, v) \geq 1$

By mapping (3), $\left\lceil\frac{f(u)+f(v)}{2}\right\rceil \geq n$.

Case 2: Suppose $u$ and $v$ are extreme vertices

As there are four extreme points in $T L G(n)$, this case is partitioned into two subcases as follows.

Case 2.1: Let $u=v_{1}$ and $v=v_{2 n}$.

Then $d(u, v)=n$.

By labeling (3), $\left\lceil\frac{f(u)+f(v)}{2}\right\rceil=n-1$.

Case 2.2: Let $u=v_{n}$ and the vertex $v=v_{n+1}$,

In this case, $d(u, v)=n-1$.

By (3), $\left\lceil\frac{f(u)+f(v)}{2}\right\rceil=n+1$.

Hence, in all the cases it can be seen that the mapping (3) satisfies the radio antipodal mean labeling condition, $d(u, v)+\left\lceil\frac{f(u)+f(v)}{2}\right\rceil \geq n$.

Therefore, (3) is a valid radio antipodal mean labeling.

By the mapping (3) the vertex $v_{2 n-1}$ receive the maximum label and the label is given by, $f\left(v_{2 n-1}\right)=2 n+k, k \equiv 0 \bmod n-4$

Hence, $\operatorname{amn}(T L G(n)) \leq 2 n+k, k \equiv 0 \bmod n-4, n \geq 3$.

\section{Radio Antipodal Mean Number of Pagoda GraphS}

In this section, the upper bound of radio antipodal mean number of pagoda graphs $P G(n)$ has been obtained.

Definition 4.1. A pagoda graph is a ladder graph formed by adding a vertex $v_{a}$ in such a way that it is adjacent to the vertices $v_{1}$ and $v_{2}$. See Figure 8 .

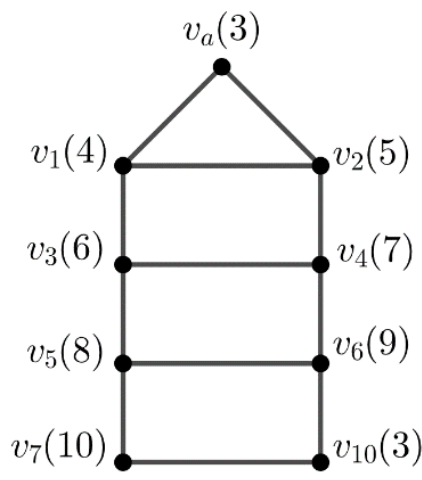

Fig. 8. $P G(4)$ and it's labeling

Remark 3. $P G(n)$ has $2 n+1$ vertices. The diameter of $P G(n)$ is $n$. The vertices $v_{1}, v_{3}, \ldots, v_{2 n-1}$ are said to be in left column (say $C_{1}$ ) of $P G(n)$, and the vertices $v_{2}, v_{4}, \ldots, v_{2 n}$ forms the right column (say $C_{2}$ ) of $P G(n)$.

Theorem 4.1. The Radio antipodal mean number $\operatorname{amn}(P G(n)) \leq 3 n-2, n \geq 2$.

Proof. Let $\left\{v_{a}, v_{1}, v_{2}, \ldots, v_{2 n}\right\}$ be the vertices of $P G(n)$. These vertices are labeled as follows.

Here, the vertices $v_{a}$ and $v_{2 n}$ are at diametric distance and so they receive the same labeling.

Label $f\left(v_{a}\right)=f\left(v_{2 n}\right)=n-1$.

The remaining vertices of $P G(n)$ are labeled by the mapping, 
$f\left(v_{i}\right)=n+i-1,1 \leq i<2 n$

Claim: Mapping (4) is a valid radio antipodal mean labeling.

In order to prove this claim, consider the following cases.

Let $u, v$ be any two vertices of $P G(n)$.

Case 1: Suppose $u=v_{a}$ and $v=v_{2 i-1}, 1 \leq i \leq n$

Then by mapping (4), $\left\lceil\frac{f(u)+f(v)}{2}\right\rceil \geq n$ and $d(u, v) \geq 1$.

Case 2: Suppose $u=v_{a}$ and $v=v_{2 i}, 1 \leq i \leq n$

The proof is similar to Case 1 .

Case 3: Suppose $u=v_{2 i-1}$ and $v=v_{2 i}, 1 \leq i \leq n-1$.

Then by mapping (4), $\left.\mid \frac{f(u)+f(v)}{2}\right\rceil \geq n$ and $d(u, v)=1$.

Case 4: Suppose $u=v_{2 i}$ and $v=v_{2 i+1}, 1 \leq i \leq n-1$.

Then by (4) $\left\lceil\frac{f(u)+f(v)}{2}\right\rceil>n$ and $d(u, v)>1$.

Case 5: Suppose $u=v_{2 i}$ and $v=v_{2 j}, i \neq j, 1 \leq i, j \leq n$

Then by (4) $\left\lceil\frac{f(u)+f(v)}{2}\right] \geq \operatorname{nand} d(u, v) \geq 1$.

Case 6: Suppose $u=v_{2 i-1}$ and $v=v_{2 j-1}, i \neq j, 1 \leq i, j \leq n$

The proof is similar to Case 4 .

Case 7: Suppose $u=v_{a}$ and $v=v_{2 n}$

Then by our assumption, $\left\lceil\frac{f(u)+f(v)}{2}\right\rceil \geq n-1$ and $d(u, v)=n$.

Hence, in all the cases it can be seen that $d(u, v)+\left\lceil\frac{f(u)+f(v)}{2}\right\rceil \geq n$.

Therefore, (4) is a valid radio antipodal mean labeling.

Here, by (4) the vertex $f\left(v_{2 n-1}\right)$ receives the maximum label and here it's label is given by,

$f\left(v_{2 n-1}\right)=3 n-2$.

Therefore, $\operatorname{amn}(P G(n)) \leq 3 n-2, n \geq 2$.

\section{CONCLUSION}

In Communication Engineering, assigning radio frequency to an arbitrary network is an interesting and challenging problem. In this paper, we have obtained the Radio antipodal mean number for certain types of ladder graphs. It is observed that the span of any network is minimized by using radio antipodal mean labeling in comparison to radio labeling, radio mean labeling and radio antipodal labeling. This work can be further extended to other types of communication networks also.

\section{REFERENCES}

[1] Ahmad Ali and RuxandraMarinescu-Ghemeci, "radio labeling of some ladder-related graphs", Math. Rep.(Bucur.) Vol. 19 Issue 69, pp. 107-119,2017

[2] Albert William, Charles Robert Kenneth, "Radio Antipodal Number of Certain Graphs", J.Comp. Math.Sci, ISSN:2319 - 8133., pp. 868-872, 2011

[3] D Antony Xavier, R C Thivyarathi, "Radio Antipodal Mean Number of Certain Graphs", International Journal of Mathematics Trends and Technology (IJMTT), ISSN:2231-5373, Vol 54, pp. 467-470, 2018

[4] Charles Robert Kenneth, Albert William, R.C. Thivyarathi, "Radio Antipodal Number of Circulant Graphs", International Journal of Engineering Research Technology, Vol. 2, 2013 
[5] Gary Chartrand, David Erwin, and P Zhang, "Radio labeling of Graphs", Bull. Inst. Combin. Appl, Vol. 33, pp. $77-85,2001$

[6] Gary Chartrand, David Erwin, and P Zhang, "Radio antipodal colorings of graphs", Math. Bohem,Vol. 127 Issue 1, pp. 57-69, 2002.

[7] Justie Su-Tzu Juan, and Daphne Der-Fen Liu, “Antipodal Labelings for Cycles”, Ars Comb., Vol. 103, pp. 81-96, 2012.

[8] M Kchikech, R Khennoufa and O Togni, "Linear and cyclic radio k-labelings of trees", DiscussionesMathematicaeGraph Theory, Vol. 130 Issue 1, pp. 105-123, 2007

[9] R Khennoufa, O Togni, “A note on radio antipodal colourings of paths”, Mathematica Bohemica, Vol. 130 Issue 3 , pp. 277-282, 2005.

[10] A Lourdusamy, F Patrick, "Vertex equitable labeling for ladder and snake related graphs", Journal of Prime Research in Mathematics, Vol. 13, pp. 01-07, 2017

[11] L Saha and P Panigrahi, "Antipodal number of some powers of cycles”, Discrete Mathematics, Vol. 312 Issue 9, pp. 1550-1557, 2012

[12] Shin Min Kang, SaimaNazeer, ImranaKousar, WaqasNazeer, and Young ChelKwun, "Multi-level and antipodal labelings for certain classes of circulant graphs”, J. Nonlinear Sci. Appl, Vol. 9, pp. 2832-2845, 2016

[13] J. Van den Heuvel, R. A. Leese, and M. A. Shepherd. "Graph labeling and radio channel assignment", Journal of Graph Theory, Vol. 29 Issue 4,pp. 263-283, 1998.

[14] William K. Hale, "Frequency Assignment: Theory and Applications", Proceedings of the IEEE, Vol. 68 Issue 12, pp. 1497-1514, 1980. 